\title{
Método experimental para identificar el nivel de atención en las personas
}

\section{An experimental method to identify the level of attention in people}

\author{
Alfredo García Suárez ${ }^{1}$ (D), Juan Manuel Gonzalez-Calleros ${ }^{1}$ (D), Amparo Palomino ${ }^{1}$ \\ ${ }^{1}$ Benemérita Universidad Autónoma de Puebla, Puebla, México. \\ alfredo.garcias@alumno.buap.mx,juan.gonzalez@correo.buap.mx, ampalomino@gmail.com
}

(Recibido: 28 julio 2021; aceptado: 9 septiembre 2021; Publicado en Internet: 1 diciembre 2021)

\begin{abstract}
Resumen. El nivel de atención de un estudiante en su entorno escolar está relacionado con diferentes variables fisiológicas del cuerpo humano. El estudio de las señales fisiológicas relacionadas con el nivel de atención como ondas cerebrales, frecuencia cardíaca, entre otros, ha revelado grandes avances en los últimos tiempos. En este trabajo se presenta el desarrollo de un sistema para medir la atención en tiempo real y de forma cuantitativa. Las variables sensadas para poder determinar el nivel de atención del usuario son: Las ondas cerebrales tipo beta y dos ángulos que describen la postura corporal del estudiante. Se busca la correlación el porcentaje de atención que se obtiene de las ondas cerebrales, con los ángulos obtenidos de la postura corporal a través de un análisis matemático. El coeficiente de correlación resultante denota que la postura corporal puede considerarse como un parámetro que influye en el nivel de atención de los estudiantes.
\end{abstract}

Palabras clave: Trastorno de déficit de atención e hiperactividad, Atención, Cerebro, Postura corporal, Procesos cognitivos.

\begin{abstract}
The student's level of attention in the school environment is related to different physiological variables of the body. The study of physiological signals related to attention such as brain waves, heart rate, breathing rate, sweat, sight tracking, among others, has revealed significant advances in recent times. This work presents the development of a system to measure the level of attention in real-time and quantitatively. The sensed variables to determine the user's level of attention are Beta-type brain waves and two angles that describe the student's corporal posture. The mathematical analysis describes the process to obtain the correlation between the percentages of the brain waves with the angles from the corporal posture. The resultant coefficient of correlation is in a considerable correlation interval. It denotes that the corporal posture can be considered a parameter that influences students' level of attention.
\end{abstract}

Keywords: Attention deficit hyperactivity disorder, Attention, Brain, Body posture, Cognitive processes.

Tipo de artículo: Artículo de investigación.

\section{Introducción}

La necesidad de actualización permanente de las prácticas educativas, la transferencia de conocimientos y la evolución tecnológica dinámica a las que nos enfrentamos con las tecnologías de la información y las comunicaciones en concordancia con las nuevas metodologías de enseñanza-aprendizaje, son aspectos fundamentales que aseguran el impacto de las acciones formativas futuras (Campazzo et al., 2011).

Recientemente diversas técnicas de análisis de sentimientos y de emociones se han utilizado para determinar el estado emocional y cognitivo de una persona debido a que el estado de ánimo influye directamente en el nivel de atención de las personas (Bustos López et al., 2016) y en consecuencia en el desempeño académico de los estudiantes (Aballay et al., 2015; Aymerich-Franch, 2012; HernándezSánchez \& Ortega, 2015).

Se han identificado distintos transtornos que influyen en el nivel de atención de las personas como se presenta en Campazzo et al. (2013) y Aymerich-Franch (2012), tanto en la infancia como en la edad adulta (Asiry et al., 2015). Uno de los trastornos más reconocidos es el Transtorno por Déficit de Atención e Hiperactividad (TDAH) y generalmente se diagnostica por primera vez en la infancia, aunque los síntomas persisten en la adolescencia e incluso hasta la edad adulta (Centers for Disease Control and Prevention, 
2021; Huh \& Ackerman, 2010). Algunas formas utilizadas para saber si una persona presenta TDAH (Frutos-Pascual et al., 2014; Sonne et al., 2016) son a través de: cuestionarios, pruebas intelectuales, tipos de comportamiento, diagnósticos médicos, entre otras (Anuradha et al., 2010; Weisberg et al., 2014). Estas pruebas requieren un largo período de tiempo donde se realiza una observación y un proceso de análisis para obtener un diagnóstico.

Para conocer el grado de afectación que produce el TDAH es necesario que las personas tengan herramientas que puedan proporcionar una retroalimentación del porcentaje de atención al ejecutar una tarea específica (Jacklyn \& Ravichandran, 2009). Actualmente existe una variedad de dispositivos comerciales que proporcionan cuantitativamente el nivel de concentración, meditación, relajación y atención (Tan \& Nijholt, 2010), pero en algunos casos se logran de manera invasiva, afectando la respuesta del usuario y en consecuencia el diagnóstico final.

Las ondas cerebrales son la variable fisiológica relacionada directamente con el nivel de atención del usuario y la postura corporal es la variable fisiológica que se consigue correlacionar con el nivel de atención (GEO Tutoriales, 2015), por medio del análisis matemático presentado en este trabajo.

Este sistema tiene un subsistema de retroalimentación (Mandryk et al., 2013; McLaren \& Antle, 2017) físico (Horii et al., 2017) y virtual (Zuckerman et al., 2016) el cual está representado a través de un semáforo. El cambio de color del semáforo indica un cambio brusco en el porcentaje de atención del usuario. Este cambio puede ser ascendente o descendente, dependiendo de la diferencia entre el valor actual y el valor anterior. Los sensores utilizados en este sistema son colocados de forma superficial en el usuario, con el propósito de estimular una respuesta natural y generar un manejo no invasivo en el desarrollo de la experimentación.

\section{Variables fisiológicas relacionadas con el nivel de atención}

Las variables fisiológicas utilizadas en este trabajo para calcular el nivel de atención son: las ondas cerebrales y la postura corporal. Las ondas cerebrales están directamente relacionadas con el porcentaje de atención cuando alcanzan un rango de frecuencia de $14 \mathrm{~Hz}$ a $26 \mathrm{~Hz}$, que pertenece a las ondas tipo beta (Torres et al., 2016). A partir del porcentaje de atención y los ángulos de la postura corporal obtenidos, se realiza un análisis matemático para encontrar el grado de correlación entre estas dos variables y determinar si la postura corporal influye de una manera significativa en el nivel de atención del usuario.

En la literatura, la variable fisiológica más utilizada para medir el nivel de atención en las personas son las ondas cerebrales (Tan \& Nijholt, 2010), estas se obtienen a través de sensores implementados en forma de diademas. Para este trabajo es utilizado el dispositivo MindWave del fabricante Neurosky.

\subsection{Ondas cerebrales}

Las señales obtenidas a través de un electroencefalograma (EEG) se pueden clasificar en 5 tipos de ondas con diferentes características. Específicamente, las ondas beta y gamma juntas se han asociado con atención, percepción y cognición (Guin \& Bikash Baishya, 2013; Torres et al., 2016).

- Ondas Beta: están en el rango de frecuencia entre $14 \mathrm{~Hz}$ y $26 \mathrm{~Hz}$, pero a menudo se dividen en beta baja y beta alta para un análisis más específico. Las ondas beta son pequeñas y rápidas. Están asociadas con la concentración focalizada. Cuando el movimiento es resistido o suprimido o, al resolver una tarea matemática hay un aumento en la actividad de las ondas beta. Un estado de pánico también puede causar un aumento en el nivel de ondas beta.

- Ondas Gamma: estas ondas están en el rango de frecuencias mayor de $30 \mathrm{~Hz}$. Su amplitud es muy pequeña, y su ocurrencia es rara, por lo que están relacionados con ciertas enfermedades del cerebro. Se cree que reflejan el mecanismo de la conciencia.

\subsection{Postura corporal}

La postura del cuerpo se define de acuerdo con los tres planos en el espacio, siendo ideal cuando refleja la simetría de los movimientos. La pérdida de verticalidad pasa por una adaptación de los diferentes segmentos 
del cuerpo con respecto a los tres planos del espacio. La actitud natural no es consciente ni voluntaria; eso es una forma de reacción debido a un estímulo constante: la gravedad (Dorbessan \& Rodriguez, 2004).

La postura no es solo un estado o una posición estables, sino es una unidad dinámica de movimiento, en la cual los brazos de palanca y los ángulos de las articulaciones son armónicos, las articulaciones pueden desarrollar un efecto de amortiguación. La postura es una forma fisiológica de usar el cuerpo.

El concepto del centro de gravedad del cuerpo es básico para analizar cualquier posición de descanso o movimiento. Es un punto ubicado en el centro exacto de la masa corporal. Su ubicación tiene pequeñas variaciones según la constitución del cuerpo, también en el mismo sujeto se mueve hacia arriba, hacia abajo o hacia los lados, de acuerdo con los cambios en posición de los segmentos orgánicos durante la actividad.

El objeto se comporta como si toda su masa estuviera centrada en ese punto, aunque en realidad, el cuerpo humano está formado por varios segmentos móviles, cada uno de los cuales tiene su propio centro. Sin embargo, cuando se evalúa la alineación de la postura erguida, se considera el cuerpo entero como un todo. El centro de gravedad se encuentra en el $55 \%$ de la altura del sujeto, por encima de la segunda vértebra sacra (Dorbessan \& Rodriguez, 2004).

El mantenimiento o recuperación de la postura normal se produce por medio de reacciones reflejas en las cuales participan receptores, vías nerviosas, centros nerviosos, reflejos miotáticos, actividad muscular tónica y fásica. Estas reacciones son: reacciones de sostén tónico-estáticas, reacciones de adaptación postural, reacciones de enderezamiento, y reacciones de equilibrio o tónico- cinéticas.

Las reacciones de sostén tónico-estáticas se producen para mantener la postura normal. Incluyen reacciones reflejas locales (originadas por el mismo miembro) y segmentarias (reacciones cruzadas). Mantienen el cuerpo erguido en contra de la fuerza de gravedad.

\subsection{Descripción del sistema de medición de atención}

En el desarrollo del sistema de adquisición de datos inicialmente solo se consideraron las ondas cerebrales como única variable de medición. Posteriormente se incluye la segunda variable que es la postura corporal. Este sistema utiliza el principio de control denominado lazo cerrado. Esta técnica se basa en una retroalimentación aproximada de tiempo real. La Figura 1 ilustra el diagrama de los elementos del sistema de reconocimiento de nivel de atención utilizando la técnica de control en lazo cerrado.

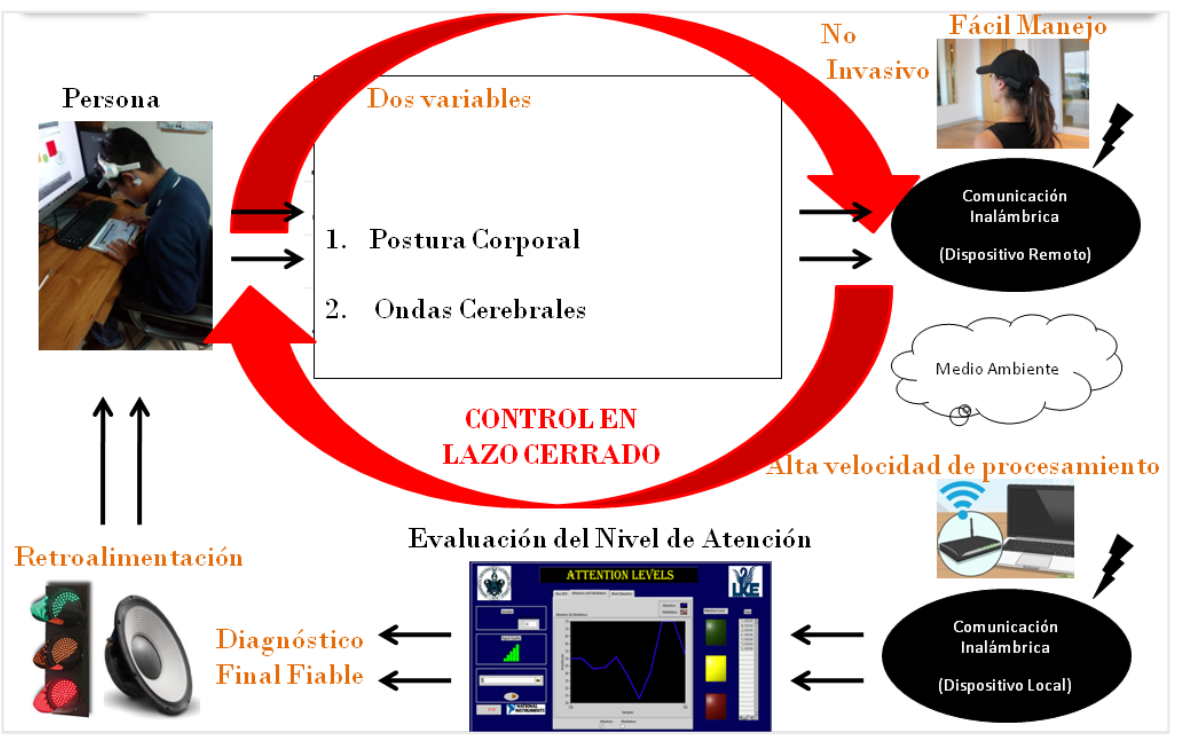

Figura 1. Sistema de identificación de nivel de atención en personas.

El funcionamiento del sistema de medición de atención inicia con las recolección de datos de las dos variables fisiológicas seleccionadas. Esta información es transmitida de manera inalámbrica de un dispositivo remoto a un dispositivo local. Posteriormente esos datos son almacenados en vectores y procesados para obtener de forma cuantitativa el porcentaje del nivel de atención del usuario en cada tiempo de muestreo. Finalmente con base al porcentaje obtenido se proporciona una retroalimentación visual en 
forma de semáforo para poder ejercer alguna acción para mantener o estimular el grado de concentración del usuario.

\section{Pruebas experimentales}

El experimento consistió en aplicar a los estudiantes una prueba para identificar colores, la cual fue obtenida de los demos de la compañía Brain $H Q$ y se explica detalladamente en (Garcia et al., 2019). El entorno grafico de la prueba de colores se muestra en la Figura 2.

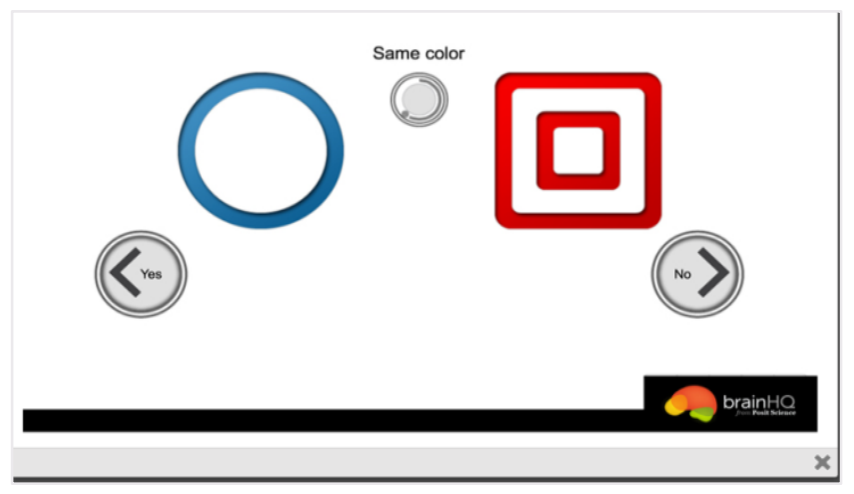

Figura 2. Entorno grafico de la prueba “same color” de la compañía Brain HQ.

La prueba fue aplicada a una muestra de 22 estudiantes de nivel primaria con TDAH diagnosticado y a una muestra de 11 estudiantes de nivel primaria sin TDAH cuyas edades oscilaron en el rango de 6 a 12 años. Las muestras fueron capturadas cada 500 milisegundos y los elementos utilizados fueron: 1 tablet (para reproducir la prueba de identificación de colores) utilizada por el usuario, 1 laptop (para monitorear los datos leídos en tiempo real a través de una interfaz gráfica) utilizada por un observador, una banda para medir la postura corporal, y una diadema de ondas cerebrales (MindWave). El desarrollo práctico del experimento se muestra en la Figura 3.

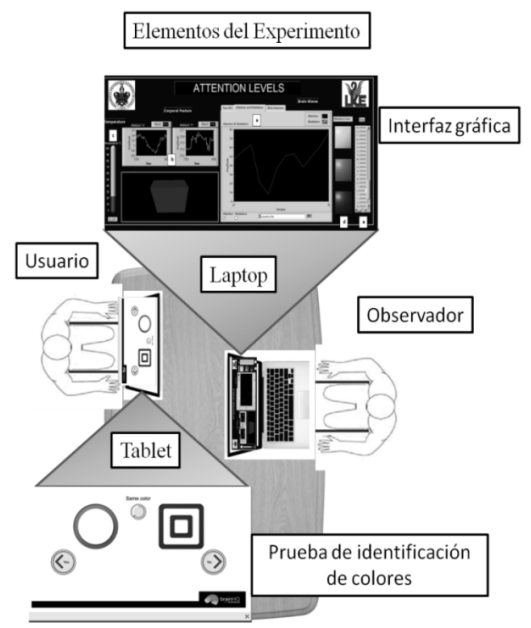

Figura 3. Diagrama de los elementos del experimento.

La recolección de muestras se llevó a cabo utilizando el dispositivo comercial MindWave de la empresa Neurosky para detectar el nivel de atención sobre las ondas cerebrales tipo beta como se describe en Garcia et al. (2019). El sensor MPU-6050 implementado en un chaleco se utilizó para medir la postura corporal a través de los ángulos obtenidos en los estudiantes de una escuela primaria en México. El sensor MPU-6050 se colocó inicialmente en un chaleco para poder recolectar las muestras de la postura corporal. El ajuste del 
chaleco por parte de los estudiantes no era práctico para su talla, esto implicaba un tiempo prolongado en la recolección de muestras, por lo tanto, fue reemplazado por una banda de ajuste, la cual resulto de fácil manejo y mejor acoplamiento a diferentes tallas.

La orientación del sensor MPU-6050 con respecto al sistema tridimensional (X, Y, Z) se adaptó de forma que el eje $\mathrm{Z}$ fuera perpendicular a la superficie de la tierra. Los ángulos medidos están formados por los movimientos alrededor del eje $\mathrm{X}$ y los movimientos alrededor del eje $\mathrm{Y}$. La causa es porque en estos ángulos está presente el soporte de las reacciones tónico-estáticas. Estas reacciones ocurren para mantener una postura normal o recta, mantienen el cuerpo erguido en contra de la fuerza de gravedad. Una mala postura hace que el usuario requiera un equilibrio neuromotor en relación con su neuro-eje lo cual tiene un impacto directo en su nivel de atención y concentración (Dorbessan \& Rodriguez, 2004). La postura del usuario es paralela al eje $\mathrm{Z}$ y relacionada con el centro de gravedad del cuerpo porque es el punto para analizar cualquier posición ya sea en reposo o en movimiento.

El análisis de datos para encontrar la correlación entre la postura corporal y el nivel de atención, comienza con la extracción de 10 características estadísticas de los ángulos de la postura corporal y una característica extraída del porcentaje de atención de las ondas cerebrales tipo beta. Finalmente se compara el desempeño de tres modelos matemáticos utilizando la técnica de regresión lineal múltiple, como se aplica en Celaya-Padilla et al. (2018) y se explica en MathWorks (2021).

El análisis de datos realizado se ilustra en la Figura 4 y tiene el siguiente estándar de componentes (MathWorks, 2021):

- Preprocesamiento. Identificación de valores atípicos y valores faltantes. Suavizado de datos para identificar posibles modelos visuales.

- Resumen o extracción de características. Cálculo de estadísticas básicas para describir la ubicación, escala y forma generales de los datos.

- Visualización: Representación gráfica de datos para identificar patrones y tendencias.

- Modelado: Descripción más detallada de las tendencias presentes en los datos, útil para predecir valores nuevos.

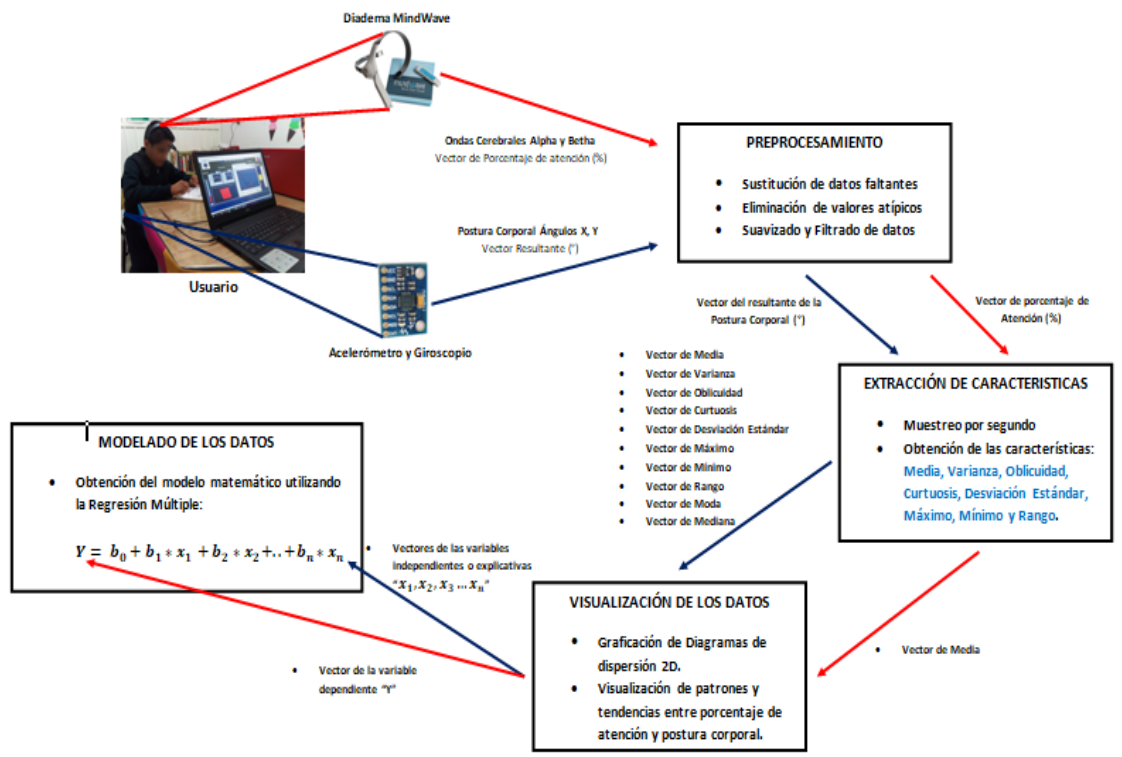

Figura 4. Análisis para obtener el modelo matemático por regresión lineal múltiple.

\section{Resultados}

En la Figura 5 se ilustran los comportamientos de los modelos de regresión lineal múltiple con mayor desempeño comparados con el porcentaje de atención original tanto para el caso de la muestra de niños con TDAH ecuación (1), como para la muestra de niños sin TDAH ecuación (2). Finalmente, en la Tabla 1 se 
presentan los resultados finales obtenidos de cada modelo matemático, utilizando como métricas de medición el coeficiente de correlación $\left(\rho_{x y}\right)$ y el coeficiente de determinación $\left(\rho_{x y}{ }^{2}\right)$ para cada caso.

$$
\begin{gathered}
Y=-610.9+328.3 x_{1}-108.2 x_{2}+233 x_{3}+471.1 x_{4}-816.7 x_{5}+6521.1 x_{6}-6476.1 x_{7} \\
-5985.9 x_{8}+0 x_{9}-375.6 x_{10} \\
Y=-639+460 x_{1}+109 x_{2}-35 x_{3}+522 x_{4}-1797 x_{5}-48559 x_{6}+48548 x_{7}+49324 x_{8}+0 x_{9} \\
-447 x_{10}
\end{gathered}
$$

Donde:

$Y$ : Es el vector de porcentaje de atención calculado a partir de las características extraídas de la postura corporal.

$x_{1}$ : Es el vector de la característica "media" extraída de la postura corporal.

$x_{2}$ : Es el vector de la característica "varianza" extraída de la postura corporal.

$x_{3}$ : Es el vector de la característica "oblicuidad" extraída de la postura corporal.

$x_{4}$ : Es el vector de la característica "curtuosis" extraída de la postura corporal.

$x_{5}$ : Es el vector de la característica "desviación estándar" extraída de la postura corporal.

$x_{6}$ : Es el vector de la característica "máximo" extraída de la postura corporal.

$x_{7}$ : Es el vector de la característica "mínimo" extraída de la postura corporal.

$x_{8}$ : Es el vector de la característica "rango" extraída de la postura corporal.

$x_{9}$ : Es el vector de la característica "moda" extraída de la postura corporal.

$x_{10}$ : Es el vector de la característica "mediana" extraída de la postura corporal.
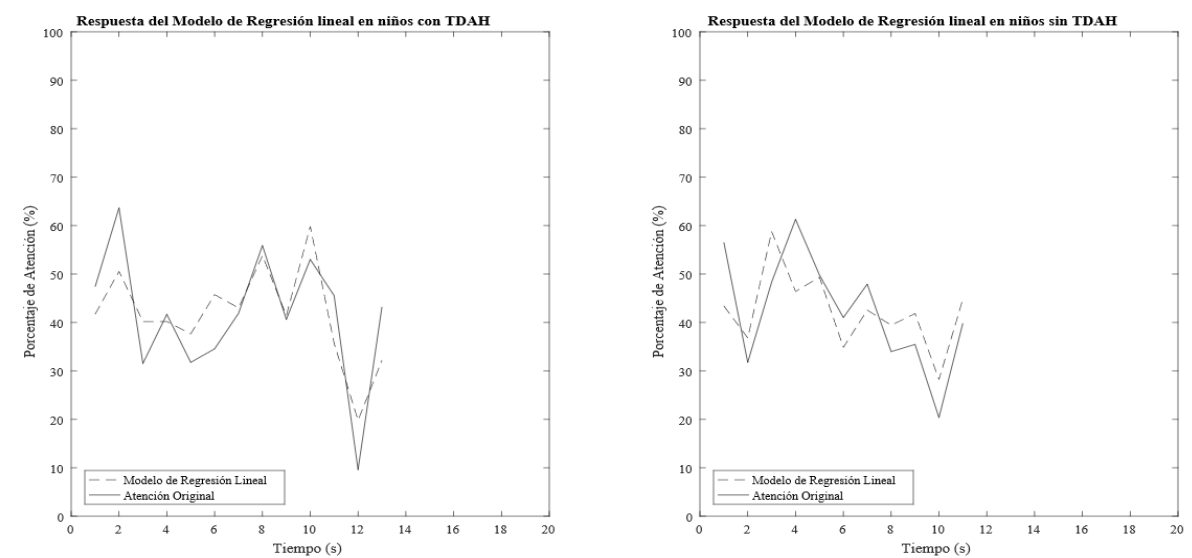

Figura 5. Respuesta de los modelos de Regresión Lineal.

Tabla 1. Coeficientes de Correlación $\left(\boldsymbol{\rho}_{\mathbf{x y}}\right)$ y de determinación $\left(\boldsymbol{\rho}_{\mathbf{x y}}{ }^{2}\right)$ obtenidos para cada modelo de regresión lineal múltiple.

\begin{tabular}{|c|c|c|c|c|}
\hline \multicolumn{2}{|c}{$\begin{array}{c}\text { Coeficiente de } \\
\text { correlación } \\
\text { Modelo }\end{array}$} & $\begin{array}{c}\text { Coeficiente de } \\
\text { correlación } \\
\left(\rho_{x y}\right) \text { en niños con } \\
\text { TDAH }\end{array}$ & $\begin{array}{c}\text { Coeficiente de } \\
\text { Teterminación }\end{array}$ & $\begin{array}{c}\text { Coeficiente de } \\
\text { determinación }\end{array}$ \\
\hline $\begin{array}{c}\text { Regresión Lineal } \\
\text { Múltiple }\end{array}$ & 0.7315 & 0.4766 & 0.5350 & 0.2271 \\
\hline $\begin{array}{c}\text { Regresión Lineal } \\
\text { Ajuste Fit }\end{array}$ & 0.6760 & 0.4701 & 0.4569 & 0.2209 \\
\hline
\end{tabular}




\section{Conclusiones}

El coeficiente de correlación $\rho_{x y}$ puede variar de -1 a 1 , donde el signo indica la dirección de la correlación y el valor numérico, la magnitud de la correlación. En este contexto se puede decir que los coeficientes de correlación obtenidos tanto para el caso de los niños con TDAH $\left(\rho_{x y}=0.7315\right)$, como para el caso de los niños sin TDAH $\left(\left(\rho_{x y}=0.4766\right)\right.$, están en el intervalo de correlación positiva considerable, descritos en GEO Tutoriales (2015).

La principal desventaja del "Modelo de regresión lineal" obtenido, es el uso de 9 de las 10 características extraídas lo cual eleva el coste computacional al realizar la predicción de los datos.

Como trabajo futuro se debe realizar una experimentación con diferentes poblaciones que tengan las mismas características empleadas en este trabajo, con el objetivo de obtener el porcentaje de atención a partir de los modelos propuestos y comprobar de forma experimental las variaciones en los coeficientes de correlación obtenidos. Además, se debe aplicar la prueba a una mayor población para validar el comportamiento de los modelos descritos en este trabajo, ya que a mayor población menor variación en el error obtenido. Finalmente se propone utilizar otras estrategias de modelado, basadas en el dominio de la frecuencia de las ondas Beta y Gamma.

\section{Declaración de conflicto de intereses}

Los autores declaran no tener conflicto de intereses con respecto a la investigación, autoría o publicación de este artículo.

\section{Financiación}

Los autores no recibieron apoyo financiero para la investigación, autoría y/o publicación de este artículo.

\section{ORCID iD}

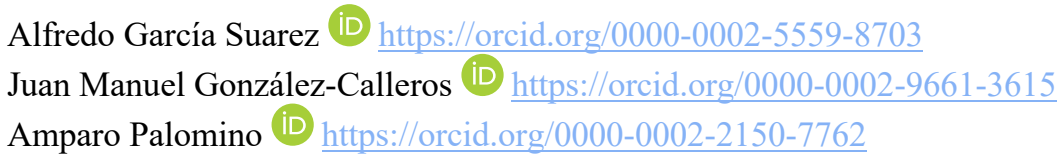

\section{Referencias}

Aballay, L. N., Aciar, S., \& Reategui, E. B. (2015). Propuesta de un método para detección de emociones en e-learning. Argentine Symposium on Artificial Intelligence (ASAI 2015) - JAIIO 44 (Rosario, 2015), 121-128. http://sedici.unlp.edu.ar/handle/10915/52106

Anuradha, J., Tisha, Ramachandran, V., Arulalan, K. v., \& Tripathy, B. K. (2010). Diagnosis of ADHD using SVM algorithm. Proceedings of the Third Annual ACM Bangalore Conference on - COMPUTE '10. https://doi.org/10.1145/1754288.1754317

Asiry, O., Shen, H., \& Calder, P. (2015, September 28). Extending Attention Span of ADHD Children through an Eye Tracker Directed Adaptive User Interface. Proceedings of the ASWEC 2015 24th Australasian Software Engineering Conference. https://doi.org/10.1145/2811681.2824997

Aymerich-Franch, L. (2012). Los juegos en entornos virtuales como herramientas de aprendizaje: estudio de la respuesta emocional de los participantes. Sphera Pública, 12, 183-197.

Bustos López, M., Hernández Montes, A. J., Vásquez Ramírez, R., Alor Hernández, G., Zatarain Cabada, R., \& Barrón Estrada, M. L. (2016). EmoRemSys: Sistema de recomendación de recursos educativos basado en detección de emociones. RISTI - Revista Ibérica de Sistemas e Tecnologias de Informação, 1(17), 80-95. https://doi.org/10.17013/risti.17.80-95 
Campazzo, E. N., Martínez, M., Guzmán, A. E., \& Agüero, A. L. (2011). Entornos Virtuales de Aprendizaje integrado a tecnología móvil y detección de emociones. XIII Workshop de Investigadores En Ciencias de La Computación. http://sedici.unlp.edu.ar/handle/10915/19943

Campazzo, E. N., Martínez, M., Guzmán, A. E., \& Agüero, A. L. (2013). Desarrollo de interface de detección de emociones para su utilización en redes sociales y entornos virtuales de aprendizaje. $X V$ Workshop de Investigadores En Ciencias de La Computación, 973-977.

Celaya-Padilla, J., Galván-Tejada, C., López-Monteagudo, F., Alonso-González, O., Moreno-Báez, A., MartínezTorteya, A., Galván-Tejada, J., Arceo-Olague, J., Luna-García, H., \& Gamboa-Rosales, H. (2018). Speed Bump Detection Using Accelerometric Features: A Genetic Algorithm Approach. Sensors, 18(2). https://doi.org/10.3390/s18020443

Centers for Disease Control and Prevention. (2021). What is ADHD? https://www.cdc.gov/ncbddd/adhd/facts.html

Dorbessan, L., \& Rodriguez, C. A. (2004). La Postura Corporal en el deporte simetrico y asimetrico.

Frutos-Pascual, M., García Zapirain, B., \& Camara Buldian, K. (2014). Adaptive Cognitive Rehabilitation Interventions based on Serious Games for Children with ADHD using Biofeedback Techniques: Assessment and Evaluation. Proceedings of the 8th International Conference on Pervasive Computing Technologies for Healthcare, 321324. https://doi.org/10.4108/icst.pervasivehealth.2014.255249

Garcia, A., Gonzalez, J. M., \& Palomino, A. (2019). Data Acquisition System for the Monitoring of Attention in People and Development of Interfaces for Commercial Devices. In V. Agredo-Delgado \& P. Ruiz (Eds.), HumanComputer Interaction. HCI-COLLAB 2018. Communications in Computer and Information Science (Vol. 847). Springer, Cham. https://doi.org/10.1007/978-3-030-05270-6_ 7

GEO Tutoriales. (2015). Cómo se relaciona el Coeficiente de Correlación de Pearson (r) y el Coeficiente de Determinación $r$ Cuadrado $\left(r^{2}\right)$. https://www.gestiondeoperaciones.net/estadistica/como-se-relaciona-elcoeficiente-de-correlacion-de-pearson-r-y-el-coeficiente-de-determinacion-r-cuadrado-r $\%$ C2\%B2/

Guin, A., \& Bikash Baishya, B. (2013). Brain Controlled Wheelchair using LabVIEW.

Hernández-Sánchez, A. M., \& Ortega, J. A. (2015). Aprendizaje Electrónico Afectivo: un modelo Innovador para Desarrollar una Acción Tutorial Virtual de Naturaleza Inclusiva. Formación Universitaria, 8(2). https://doi.org/10.4067/S0718-50062015000200004

Horii, T., Nagai, Y., \& Asada, M. (2017, October 27). Active Perception based on Energy Minimization in Multimodal Human-robot Interaction. Proceedings of the 5th International Conference on Human Agent Interaction. https://doi.org/10.1145/3125739.3125757

Huh, J., \& Ackerman, M. S. (2010, April 10). Exploring social dimensions of personal information management with adults with AD/HD. CHI '10 Extended Abstracts on Human Factors in Computing Systems. https://doi.org/10.1145/1753846.1754044

Jacklyn, H. Q., \& Ravichandran, S. (2009). Motivating Children with Attention Deficiency Disorder Using Certain Behavior Modification Strategies. In C. T. Lim \& J. C. H. Goh (Eds.), 13th International Conference on Biomedical Engineering. IFMBE Proceedings (Vol. 23, pp. 1057-1060). Springer Berlin Heidelberg. https://doi.org/10.1007/978-3-540-92841-6

Mandryk, R. L., Dielschneider, S., Kalyn, M. R., Bertram, C. P., Gaetz, M., Doucette, A., Taylor, B. A., Orr, A. P., \& Keiver, K. (2013, June 24). Games as neurofeedback training for children with FASD. Proceedings of the 12th International Conference on Interaction Design and Children. https://oi.org/10.1145/2485760.2485762

MathWorks. (2021). Data Analysis. https://es.mathworks.com/help/matlab/learn_matlab/data-analysis.html?lang=en

McLaren, E.-S., \& Antle, A. N. (2017, June 27). Exploring and Evaluating Sound for Helping Children Self-Regulate with a Brain-Computer Application. Proceedings of the 2017 Conference on Interaction Design and Children. https://doi.org/10.1145/3078072.3084299

Sonne, T., Marshall, P., Obel, C., Thomsen, P. H., \& Grønbæk, K. (2016). An assistive technology design framework for ADHD. Proceedings of the 28th Australian Conference on Computer-Human Interaction - OzCHI '16. https://doi.org/10.1145/3010915.3010925

Tan, D. S., \& Nijholt, A. (2010). Brain-Computer Interfaces. Applying our Minds to Human-Computer Interaction. Springer London. https://doi.org/10.1007/978-1-84996-272-8

Torres, F., Sánchez, C., \& Palacios Baus, K. (2016). Adquisición y análisis de señales cerebrales utilizando el dispositivo MindWave. Maskana, 5, 83-93.

Weisberg, O., GalOz, A., Berkowitz, R., Weiss, N., Peretz, O., Azoulai, S., KoplemanRubin, D., \& Zuckerman, O. (2014, June 17). TangiPlan: designing an assistive technology to enhance executive functioning among children with ADHD. Proceedings of the 2014 Conference on Interaction Design and Children. https://doi.org/10.1145/2593968.2610475

Zuckerman, O., Hoffman, G., Kopelman-Rubin, D., Klomek, A. B., Shitrit, N., Amsalem, Y., \& Shlomi, Y. (2016, February 14). KIP3: Robotic Companion as an External Cue to Students with ADHD. Proceedings of the TEI '16: Tenth International Conference on Tangible, Embedded, and Embodied Interaction. https://doi.org/10.1145/2839462.2856535 